\title{
Biospecimen Submitted to BCR
}

National Cancer Institute

\section{Source}

National Cancer Institute. Biospecimen Submitted to BCR. NCI Thesaurus. Code

C159201.

A section header about biospecimens submitted to BCR. 